$\mathbf{T}_{\text {he }} \mathbf{J}_{\text {ournal of }} \mathbf{N}_{\text {onlinear }} \mathbf{S}_{\text {ciences and }}$ Applications http://www.tjnsa.com

\title{
COMMON FIXED POINT THEOREMS IN CONE METRIC SPACES
}

\author{
AKBAR AZAM ${ }^{1}$, MUHAMMAD ARSHAD ${ }^{2}$ AND ISMAT BEG ${ }^{3, *}$
}

\begin{abstract}
We obtain sufficient conditions for existence of points of coincidence and common fixed points of three self mappings satisfying a contractive type conditions in cone metric spaces. Our results generalize several well-known recent results.
\end{abstract}

\section{INTRODUCTION AND PRELIMINARIERS}

Since the appearance of the Banach contraction mapping principle, a number of papers were dedicated to the improvement and generalization of that result. Most of these deal with the generalizations of the contractive condition (see [2, 3, 4, 7, 8, 9, 10, 11, 12, 14, 15, 16] and references there in) in metric spaces.

Guang and Zhang [5] recently introduced the concept of cone metric spaces and established some fixed point theorems for contractive type mappings in a normal cone metric space. Subsequently, some other authors [1, 6, 17] studied the existence of points of coincidence, and common fixed points of mappings satisfying a contractive type condition in cone metric spaces. Afterwards, Rezapour and Hamlbarani [13] studied fixed points theorems of contractive type mappings by omitting the assumption of normality in cone metric spaces. In this paper we obtain points of coincidence and common fixed points for three self mappings satisfying Jungck [7] type contractive condition without the assumption of normality in cone metric spaces.

First we recall Jungck's [7] theorem:

Date: Revised 05/07/09

* Corresponding author.

2000 Mathematics Subject Classification. 47H10; 54H25.

Key words and phrases. Point of coincidence; common fixed point; contractive type mapping; commuting mapping; compatible mapping; cone metric space. 
Theorem 1.1. Let $(X, \rho)$ be a complete metric space. Let $f$ be a continuous self-map on $X$ and $g$ be any self-map on $X$ that commutes with $f$. Further let $f$ and $g$ satisfy $g(X) \subseteq f(X)$ and there exists a constant $\lambda \in(0,1)$ such that for every $x, y \in X$

$$
\rho(g x, g y) \leq \lambda \rho(f x, f y)
$$

Then $f$ and $g$ have a unique common fixed point.

Sessa [16] generalized the concept of commuting mappings by calling self mappings $f, g$ on a metric space $X$, weakly commuting if and only if

$$
d(f g x, g f x) \leq d(f x, g x)
$$

for all $x \in X$. Of course commuting mappings are weakly commuting but converse is not true in general (see [16]). Afterwards, many authors obtained nice fixed point theorems by using this concept. However elementary function as simple as $f x=x^{3}, g x=2 x^{3}$ are not weakly commuting. Thus Jungck [8] and Pant [12] introduced some less restrictive concepts of compatible mappings and R-weakly commuting mappings respectively. Later on, it has been noticed that compatible mappings and R-weakly commuting mappings commute at their coincidence point. Jungck and Rhoades [11], then defined a pair $(f, g)$ of selfmappings to be weakly compatible if they commute at their coincidence point ( i.e. $f g x=g f x$ whenever $f x=g x)$.

A subset $P$ of a real Banach space $E$ is called a cone if it has following properties:

(i) $P$ is non-empty closed and $P \neq\{\mathbf{0}\}$;

(ii) $0 \leq a, b \in R$ and $x, y \in P \Rightarrow a x+b y \in P$;

(iii) $P \cap(-P)=\{\mathbf{0}\}$.

For a given cone $P \subseteq E$, we can define a partial ordering $\leq$ on $E$ with respect to $P$ by $x \leq y$ if and only if $y-x \in P$. We shall write $x<y$ if $x \leq y$ and $x \neq y$, while $x \ll y$ will stands for $y-x \in$ int $P$, where int $P$ denotes the interior of $P$.The cone $P$ is called normal if there is a number $k>0$ such that for all $x, y, \in E$,

$$
\mathbf{0} \leq x \leq y \Rightarrow\|x\| \leq \kappa\|y\| .
$$

The least positive number $\kappa$ satisfying (I) is called the normal constant of $P$. There are no normal cones with normal constant $\kappa<1$ [13]. Also [13, example 2.3] shows that there are non-normal cones.

In the following we always suppose that $E$ is a real Banach space and $P$ is a cone in $E$ with int $P \neq \emptyset$ and $\leq$ is a partial ordering with respect to $P$.

Definition 1.2. Let $X$ be a nonempty set. Suppose that the mapping $d: X \times$ $X \rightarrow E$, satisfies:

(1) $\mathbf{0} \leq d(x, y)$, for all $x, y \in X$ and $d(x, y)=\mathbf{0}$ if and only if $x=y$;

(2) $d(x, y)=d(y, x)$ for all $x, y \in X$;

(3) $d(x, y) \leq d(x, z)+d(z, y)$, for all $x, y, z \in X$. 
Then $d$ is called a cone metric on $X$, and $(X, d)$ is called a cone metric space.

Let $x_{n}$ be a sequence in $X$, and $x \in X$. If for every $c \in E$, with $\mathbf{0} \ll c$ there is $n_{0} \in \mathbb{N}$ such that for all $n>n_{0}, d\left(x_{n}, x\right) \ll c$, then $\left\{x_{n}\right\}$ is said to be convergent, $\left\{x_{n}\right\}$ converges to $x$ and $x$ is the limit of $\left\{x_{n}\right\}$.We denote this by $\lim _{n} x_{n}=x$, or $x_{n} \longrightarrow x$,as $n \rightarrow \infty$. If for every $c \in E$ with $0 \ll c$ there is $n_{0} \in \mathbb{N}$ such that for all $n, m>n_{0}, d\left(x_{n}, x_{m}\right) \ll c$, then $\left\{x_{n}\right\}$ is called a Cauchy sequence in $X$. If every Cauchy sequence is convergent in $X$, then $X$ is called a complete cone metric space. Let us recall [5] that if $P$ is a normal cone, then $x_{n} \in X$ converges to $x \in X$ if and only if $d\left(x_{n}, x\right) \rightarrow \mathbf{0}$ as $n \rightarrow \infty$. Furthermore, $x_{n} \in X$ is a Cauchy sequence if and only if $d\left(x_{n}, x_{m}\right) \rightarrow \mathbf{0}$ as $n, m \rightarrow \infty$.

A point $y \in X$ is called point of coincidence of $T, f: X \rightarrow X$ if there exists a point $x \in X$ such that $y=f x=T x$.

\section{MAIN RESULTS}

We start with a lemma, which will be required in the sequel.

Lemma 2.1. Let $X$ be a non-empty set and the mappings $S, T, f: X \rightarrow X$ have a unique point of coincidence $v$ in $X$. If $(S, f)$ and $(T, f)$ are weakly compatible, then $S, T$ and $f$ have a unique common fixed point.

Proof. Since $v$ is point of coincidence $S, T$ and $f$. Therefore, $v=f u=S u=T u$ for some $u \in X$. By weakly compatibility of $(S, f)$ and $(T, f)$ we have

$$
S v=S f u=f S u=f v \text { and } T v=T f u=f T u=f v .
$$

It implies that $S v=T v=f v=w$ (say). Then $w$ is a point of coincidence of $S, T$ and $f$. Therefore, $v=w$ by uniqueness. Thus $v$ is a unique common fixed point of $S, T$ and $f$.

Theorem 2.2. Let $(X, d)$ be a cone metric space and the mappings $S, T, f$ : $X \rightarrow X$ satisfy:

$$
d(S x, T y) \leq \lambda d(f x, f y)
$$

for all $x, y \in X$ where $0 \leq \lambda<1$.If

$$
S(X) \cup T(X) \subseteq f(X)
$$

and $f(X)$ is a complete subspace of $X$, then $S, T$ and $f$ have a unique point of coincidence. Moreover if $(S, f)$ and $(T, f)$ are weakly compatible, then $S, T$ and $f$ have a unique common fixed point.

Proof. Let $x_{0}$ be an arbitrary point in $X$. Choose a point $x_{1}$ in $X$ such that $f x_{1}=S x_{0}$. This can be done since $S(X) \subseteq f(X)$. Similarly, choose a point $x_{2}$ in $X$ such that $f x_{2}=T x_{1}$. Continuing this process and having chosen $x_{n}$ in $X$. We obtain $x_{n+1}$ in $X$ such that

$$
\begin{aligned}
& f x_{2 k+1}=S x_{2 k} \\
& f x_{2 k+2}=T x_{2 k+1}, k=0,1,2, \ldots
\end{aligned}
$$

Then,

$$
\begin{aligned}
d\left(f x_{2 k+1}, f x_{2 k+2}\right) & =d\left(S x_{2 k}, T x_{2 k+1}\right) \\
& \leq \lambda d\left(f x_{2 k}, f x_{2 k+1}\right)
\end{aligned}
$$


Similarly,

$$
\begin{aligned}
d\left(f x_{2 k+2}, f x_{2 k+3}\right) & =d\left(S x_{2 k+2}, T x_{2 k+1}\right) \\
& \leq \lambda\left(f x_{2 k+2}, f x_{2 k+1}\right)
\end{aligned}
$$

Now by induction, we obtain for each $k=0,1,2, \ldots$,

$$
d\left(f x_{2 k+2}, f x_{2 k+3}\right) \leq \lambda^{2 k+2} d\left(f x_{0}, f x_{1}\right)
$$

Let

$$
y_{n}=f x_{n}, n=0,1,2, \ldots
$$

Now for all $n$, we have

$$
\begin{aligned}
d\left(y_{n+1}, y_{n+2}\right) & \leq \lambda d\left(y_{n}, y_{n+1}\right) \\
& \leq \cdots \leq \lambda^{n+1} d\left(y_{0}, y_{1}\right)
\end{aligned}
$$

Now for any $m>n$,

$$
\begin{aligned}
d\left(y_{m}, y_{n}\right) & \leq d\left(y_{n}, y_{n+1}\right)+d\left(y_{n+1}, y_{n+2}\right)+\ldots+d\left(y_{m-1}, y_{m}\right) \\
& \leq\left[\lambda^{n}+\lambda^{n+1}+\ldots+\lambda^{m-1}\right] d\left(y_{0}, y_{1}\right) \\
& \leq\left[\frac{\lambda^{n}}{1-\lambda}\right] d\left(y_{0}, y_{1}\right)
\end{aligned}
$$

Let $\mathbf{0} \ll c$ be given. Choose $\delta>0$ such that

$$
c+\{x \in E:\|x\|<\delta\} \subseteq P .
$$

Also, choose a natural number $N_{1}$, such that

$$
\frac{\lambda^{n}}{1-\lambda} d\left(y_{0}, y_{1}\right) \in\{x \in E:\|x\|<\delta\}, \text { for all } n \geq N_{1}
$$

Then

$$
\left[\frac{\lambda^{n}}{1-\lambda}\right] d\left(y_{0}, y_{1}\right) \ll c, \text { for all } n \geq N_{1}
$$

Thus,

$$
m>n \geq N_{1 .} \Rightarrow d\left(y_{m}, y_{n}\right) \leq\left[\frac{\lambda^{n}}{1-\lambda}\right] d\left(y_{0}, y_{1}\right) \ll c,
$$

which implies that $\left\{y_{n}\right\}$ is a Cauchy sequence. Since $f(X)$ is complete, there exists $u, v \in X$ such that $y_{n} \rightarrow v=f u$. Choose a natural number $N_{2}$ such that

$$
d\left(y_{n}, v\right) \ll \frac{c}{2} \text { for all } n \geq N_{2} \text {. }
$$

Hence, for all $n \geq N_{2}$

$$
\begin{aligned}
d(f u, S u) & \leq d\left(f u, y_{2 n+2}\right)+d\left(y_{2 n+2}, S u\right) \\
& \leq d\left(v, y_{2 n+2}\right)+d\left(T x_{2 n+1}, S u\right) \\
& \leq d\left(v, y_{2 n+2}\right)+\lambda d\left(f x_{2 n+1}, f u\right) \\
& \leq d\left(v, y_{2 n+2}\right)+d\left(y_{2 n+1}, v\right) \ll \frac{c}{2}+\frac{c}{2}=c .
\end{aligned}
$$

Thus

$$
d(f u, S u) \ll \frac{c}{m}, \text { for all } m \geq 1 .
$$


So, $\frac{c}{m}-d(f u, S u) \in P$,for all $m \geq 1$. Since $\frac{c}{m} \rightarrow \mathbf{0}($ as $m \rightarrow \infty)$ and $P$ is closed, $-d(f u, S u) \in P$, but $P \cap(-P)=\{\mathbf{0}\}$. Therefore, $d(f u, S u)=\mathbf{0}$. Hence $f u=S u$.Similarly, by using

$$
d(f u, T u) \leq d\left(f u, y_{2 n+1}\right)+d\left(y_{2 n+1}, T u\right),
$$

we can show that $f u=T u$, it implies that $v$ is a common point of coincidence of $S, T$ and $f$ that is

$$
v=f u=S u=T u .
$$

Now we show that $f, S$ and $T$ have unique point of coincidence. For this, assume that there exists another point $v^{*}$ in $X$ such that $v^{*}=f u^{*}=S u^{*}=T u^{*}$ for some $u^{*}$ in $X$. Now,

$$
\begin{aligned}
d\left(v, v^{*}\right) & =d\left(S u, T u^{*}\right) \\
& \leq \lambda d\left(f u, f u^{*}\right) \\
& \leq \lambda d\left(v, v^{*}\right) .
\end{aligned}
$$

This implies that $v^{*}=v$. If $(S, f)$ and $(T, f)$ are weakly compatible,by Lemma 2.1, $S, T$ and $f$ have a unique common fixed point.

Theorem 2.3. Let $(X, d)$ be a cone metric space and the mappings $S, T, f$ : $X \rightarrow X$ satisfy:

$$
d(S x, T y) \leq \lambda[d(f x, S x)+d(f y, T y)]
$$

for all $x, y \in X$ where $0 \leq \lambda<\frac{1}{2}$.If

$$
S(X) \cup T(X) \subseteq f(X)
$$

and $f(X)$ is a complete subspace of $X$, then $S, T$ and $f$ have a unique point of coincidence. Moreover, if $(S, f)$ and $(T, f)$ are weakly compatible, then $S, T$ and $f$ have a unique common fixed point.

Proof. Let $x_{0}$ be an arbitrary point in $X$. Define a sequence of points in $X$, as in Theorem 4, given by the rule:

$$
\begin{aligned}
& f x_{2 k+1}=S x_{2 k} \\
& f x_{2 k+2}=T x_{2 k+1}, k=0,1,2, \ldots
\end{aligned}
$$

Then,

$$
\begin{aligned}
d\left(f x_{2 k+1}, f x_{2 k+2}\right) & =d\left(S x_{2 k}, T x_{2 k+1}\right) \\
& \leq \lambda\left[d\left(f x_{2 k}, S x_{2 k}\right)+\left(f x_{2 k+1}, T x_{2 k+1}\right)\right] \\
& \leq \lambda\left[d\left(f x_{2 k}, f x_{2 k+1}\right)+\left(f x_{2 k+1}, f x_{2 k+2}\right)\right] \\
& \leq \frac{\lambda}{1-\lambda}\left[d\left(f x_{2 k}, f x_{2 k+1}\right)\right] .
\end{aligned}
$$

Similarly it can be shown that

$$
d\left(f x_{2 k+2}, f x_{2 k+3}\right)=\frac{\lambda}{1-\lambda}\left(f x_{2 k+1}, f x_{2 k+2}\right) .
$$


Now by induction,we obtain for each $k=0,1,2, \ldots$,

$$
\begin{aligned}
d\left(f x_{2 k+1}, f x_{2 k+2}\right) & \leq \frac{\lambda}{1-\lambda} d\left(f x_{2 k}, f x_{2 k+1}\right) \\
& \leq\left[\frac{\lambda}{1-\lambda}\right]^{2} d\left(f x_{2 k-1}, f x_{2 k}\right) \\
& \leq \cdots \leq\left[\frac{\lambda}{1-\lambda}\right]^{2 k+1} d\left(f x_{0}, f x_{1}\right)
\end{aligned}
$$

and

$$
d\left(f x_{2 k+2}, f x_{2 k+3}\right) \leq\left[\frac{\lambda}{1-\lambda}\right]^{2 k+2} d\left(f x_{0}, f x_{1}\right)
$$

Let

$$
\left[\frac{\lambda}{1-\lambda}\right]=h \text { and } y_{n}=f x_{n}, n=0,1,2, \ldots
$$

Now for all $n=0,1,2, \ldots$ We have

$$
\begin{aligned}
d\left(y_{n+1}, y_{n+2}\right) & \leq h d\left(y_{n}, y_{n+1}\right) \\
& \leq \cdots \leq h^{n+1} d\left(y_{0}, y_{1}\right)
\end{aligned}
$$

Now for any $m>n$,

$$
\begin{aligned}
d\left(y_{m}, y_{n}\right) & \leq d\left(y_{n}, y_{n+1}\right)+d\left(y_{n+1}, y_{n+2}\right)+\ldots+d\left(y_{m-1}, y_{m}\right) \\
& \leq\left[h^{n}+h^{n+1}+\ldots+h^{m-1}\right] d\left(y_{0}, y_{1}\right) \\
& \leq\left[\frac{h^{n}}{1-h}\right] d\left(y_{0}, y_{1}\right)
\end{aligned}
$$

Let $\mathbf{0} \ll c$ be given. Choose $\delta>0$ such that

$$
c+\{x \in E:\|x\|<\delta\} \subseteq P .
$$

Also choose a natural number $N_{1}$ such that

$$
\frac{h^{n}}{1-h} d\left(y_{0}, y_{1}\right) \in\{x \in E:\|x\|<\delta\} \text {, for all } n \geq N_{1} \text {. }
$$

Then

$$
\left[\frac{h^{n}}{1-h}\right] d\left(y_{0}, y_{1}\right) \ll c, \text { for all } n \geq N_{1}
$$

Thus

$$
m>n \geq N_{1 .} \Rightarrow d\left(y_{m}, y_{n}\right) \leq\left[\frac{h^{n}}{1-h}\right] d\left(y_{0}, y_{1}\right) \ll c,
$$

which implies that $\left\{y_{n}\right\}$ is a Cauchy sequence. Since $f(X)$ is complete, there exists $u, v \in X$ such that $y_{n} \rightarrow v=f u$. Choose a natural number $N_{2}$ such that

$$
d\left(y_{n+1}, y_{n}\right) \ll \frac{c(1-\lambda)}{2 \lambda} \text { and } d\left(y_{n+1}, v\right) \ll \frac{c(1-\lambda)}{2} \text { for all } n \geq N_{2} \text {. }
$$


Hence, for all $n \geq N_{2}$

$$
\begin{aligned}
d(f u, S u) & \leq d\left(f u, y_{2 n+2}\right)+d\left(y_{2 n+2}, S u\right) \\
& \leq d\left(v, y_{2 n+2}\right)+d\left(T x_{2 n+1}, S u\right) \\
& \leq d\left(v, y_{2 n+2}\right)+\lambda\left[d(f u, S u)+d\left(f x_{2 n+1}, T x_{2 n+1}\right)\right] \\
& \leq \frac{1}{1-\lambda} d\left(v, y_{2 n+2}\right)+\frac{\lambda}{1-\lambda} d\left(y_{2 n+1}, y_{2 n+2}\right) \ll \frac{c}{2}+\frac{c}{2}=c .
\end{aligned}
$$

Thus

$$
d(f u, S u) \ll \frac{c}{m} \text {, for all } m \geq 1 .
$$

So, $\frac{c}{m}-d(f u, S u) \in P$, for all $m \geq 1$. Since $\frac{c}{m} \rightarrow \mathbf{0}($ as $m \rightarrow \infty)$ and $P$ is closed, $-d(f u, S u) \in P$ but $P \cap(-P)=\{\mathbf{0}\}$.Therefore, $d(f u, S u)=\mathbf{0}$. Hence, $f u=S u$. Similarly, by using

$$
d(f u, T u) \leq d\left(f u, y_{2 n+1}\right)+d\left(y_{2 n+1}, T u\right),
$$

we can show that $f u=T u$. It implies that $v$ is a common point of coincidence of $S, T$ and $f$ that is

$$
v=f u=S u=T u \text {. }
$$

Now we show that $f, S$ and $T$ have unique point of coincidence. For this, assume that there exists another point $v^{*}$ in $X$ such that $v^{*}=f u^{*}=S u^{*}=T u^{*}$ for some $u^{*}$ in $X$.Then,

$$
\begin{aligned}
d\left(v, v^{*}\right) & =d\left(S u, T u^{*}\right) \\
& \leq \lambda\left[d(f u, S u)+d\left(f u^{*}, T u^{*}\right)\right] \\
& \leq \lambda\left[d(v, v)+d\left(v^{*}, v^{*}\right)\right]=0
\end{aligned}
$$

It implies that Hence $v=v^{*}$. If $(S, f)$ and $(T, f)$ are weakly compatible,by Lemma 2.1, S, T and $f$ have a unique common fixed point.

Theorem 2.4. Let $(X, d)$ be a cone metric space and the mappings $S, T, f$ : $X \rightarrow X$ satisfy.

$$
d(S x, T y) \leq \lambda[d(f y, S x)+d(f x, T y)]
$$

for all $x, y \in X$ where $0 \leq \lambda<\frac{1}{2}$. If

$$
S(X) \cup T(X) \subseteq f(X)
$$

and $f(X)$ is a complete subspace of $X$, then $S, T$ and $f$ have a unique point of coincidence. Moreover, if $(S, f)$ and $(T, f)$ are weakly compatible, then $S, T$ and $f$ have a unique common fixed point.

Proof. Let $x_{0}$ be an arbitrary point in $X$. Define a sequence of points in $X$, as in Theorem 4, given by the rule:

$$
\begin{aligned}
f x_{2 k+1} & =S x_{2 k} \\
f x_{2 k+2} & =T x_{2 k+1}, k=0,1,2, \ldots
\end{aligned}
$$


Then

$$
\begin{aligned}
d\left(f x_{2 k+1}, f x_{2 k+2}\right) & =d\left(S x_{2 k}, T x_{2 k+1}\right) \\
& \leq \lambda\left[d\left(f x_{2 k+1}, S x_{2 k}\right)+\left(f x_{2 k}, T x_{2 k+1}\right)\right] \\
& \leq \lambda\left[d\left(f x_{2 k+1}, f x_{2 k+1}\right)+\left(f x_{2 k}, f x_{2 k+2}\right)\right] \\
& \leq \frac{\lambda}{1-\lambda}\left[d\left(f x_{2 k}, f x_{2 k+1}\right)\right] .
\end{aligned}
$$

Similarly, it can be shown that

$$
d\left(f x_{2 k+2}, f x_{2 k+3}\right)=\frac{\lambda}{1-\lambda}\left(f x_{2 k+1}, f x_{2 k+2}\right)
$$

Now by induction,we obtain for each $k=0,1,2, \ldots$,

$$
\begin{aligned}
d\left(f x_{2 k+1}, f x_{2 k+2}\right) & \leq \frac{\lambda}{1-\lambda} d\left(f x_{2 k}, f x_{2 k+1}\right) \\
& \leq\left[\frac{\lambda}{1-\lambda}\right]^{2} d\left(f x_{2 k-1}, f x_{2 k}\right) \\
& \leq \cdots \leq\left[\frac{\lambda}{1-\lambda}\right]^{2 k+1} d\left(f x_{0}, f x_{1}\right)
\end{aligned}
$$

and

$$
d\left(f x_{2 k+2}, f x_{2 k+3}\right) \leq\left[\frac{\lambda}{1-\lambda}\right]^{2 k+2} d\left(f x_{0}, f x_{1}\right)
$$

Let

$$
\left[\frac{\lambda}{1-\lambda}\right]=h \text { and } y_{n}=f x_{n}, n=0,1,2, \ldots
$$

Now for all $n$ we have

$$
\begin{aligned}
d\left(y_{n+1}, y_{n+2}\right) & \leq h d\left(y_{n}, y_{n+1}\right) \\
& \leq \cdots \leq h^{n+1} d\left(y_{0}, y_{1}\right)
\end{aligned}
$$

Now for any $m>n$,

$$
\begin{aligned}
d\left(y_{m}, y_{n}\right) & \leq d\left(y_{n}, y_{n+1}\right)+d\left(y_{n+1}, y_{n+2}\right)+\ldots+d\left(y_{m-1}, y_{m}\right) \\
& \leq\left[h^{n}+h^{n+1}+\ldots+h^{m-1}\right] d\left(y_{0}, y_{1}\right) \\
& \leq\left[\frac{h^{n}}{1-h}\right] d\left(y_{0}, y_{1}\right) .
\end{aligned}
$$

Let $\mathbf{0} \ll c$ be given. Choose $\delta>0$ such that

$$
c+\{x \in E:\|x\|<\delta\} \subseteq P .
$$

Also choose a natural number $N_{1}$ such that

$$
\frac{h^{n}}{1-h} d\left(y_{0}, y_{1}\right) \in\{x \in E:\|x\|<\delta\} \text {, for all } n \geq N_{1} \text {. }
$$

Then

$$
\left[\frac{h^{n}}{1-h}\right] d\left(y_{0}, y_{1}\right) \ll c, \text { for all } n \geq N_{1}
$$


Thus

$$
m>n \geq N_{1 .} \Rightarrow d\left(y_{m}, y_{n}\right) \leq\left[\frac{h^{n}}{1-h}\right] d\left(y_{0}, y_{1}\right) \ll c,
$$

it implies that $\left\{y_{n}\right\}$ is a Cauchy sequence. Since $f(X)$ is complete, there exists $u, v \in X$ such that $y_{n} \rightarrow v=f u$. Choose a natural number $N_{2}$ such that

$$
d\left(y_{n+1}, v\right) \ll \frac{c(1-\lambda)}{3} \text { for all } n \geq N_{2} .
$$

Hence, for all $n \geq N_{2}$

$$
\begin{aligned}
d(f u, S u) \leq & d\left(f u, y_{2 n+1}\right)+d\left(y_{2 n+1}, S u\right) \\
\leq & d\left(v, y_{2 n+1}\right)+d\left(T x_{2 n+1}, S u\right) \\
\leq & d\left(v, y_{2 n+1}\right)+\lambda\left[d\left(f u, T x_{2 n+1}\right)+d\left(f x_{2 n+1}, S u\right)\right] \\
\leq & d\left(v, y_{2 n+1}\right)+\lambda\left[d\left(y_{2 n+2}, v\right)+d\left(f x_{2 n+1}, v\right)+d(f u, S u)\right] \\
& \frac{1}{1-\lambda}\left[d\left(v, y_{2 n+1}\right)+\lambda\left(d\left(y_{2 n+2}, v\right)+d\left(f x_{2 n+1}, v\right)\right)\right] \\
\ll & \frac{c}{3}+\frac{c}{3}+\frac{c}{3}=c .
\end{aligned}
$$

Thus

$$
d(f u, S u) \ll \frac{c}{m} \text {, for all } m \geq 1 .
$$

So, $\frac{c}{m}-d(f u, S u) \in P$, for all $m \geq 1$. Since $\frac{c}{m} \rightarrow \mathbf{0}($ as $m \rightarrow \infty)$ and $P$ is closed, $-d(f u, S u) \in P$. But $d(f u, S u) \in P$. Therefore, $d(f u, S u)=\mathbf{0}$. Hence $f u=S u$.Similarly, by using

$$
d(f u, T u) \leq d\left(f u, y_{2 n+1}\right)+d\left(y_{2 n+1}, T u\right),
$$

we can show that $f u=T u$. Which implies that $v$ is a common point of coincidence of $S, T$ and $f$ that is

$$
v=f u=S u=T u .
$$

Then we show that $f, S$ and $T$ have unique point of coincidence For this, assume that there exists another point $v^{*}$ in $X$ such that $v^{*}=f u^{*}=S u^{*}=T u^{*}$ for some $u^{*}$ in $X$. Now,

$$
\begin{aligned}
d\left(v, v^{*}\right) & =d\left(S u, T u^{*}\right) \\
& \leq \lambda\left[d\left(f u, T u^{*}\right)+d\left(f u^{*}, S u\right)\right] \\
& \leq 2 \lambda d\left(v, v^{*}\right)
\end{aligned}
$$

It implies that $v=v^{*}$. By Lemma $2.1 S, T$ and $f$ have a unique common fixed point if $(S, f)$ and $(T, f)$ are weakly compatible.

\section{Conclusion}

The particular cases (when $f=I$ the identity maps or $S=T$ ) of our results generalize the theorems 1, 3, 4 of [5] and theorems. 2.3, 2.6, 2.7 of [13]. Moreover our results also generalize theorems 2.1, 2.3, 2.4.of [1] even in the case when $S=T$, since $(X, d)$ is not assumed to have a normal cone $P$. 


\section{REFERENCES}

[1] M. Abbas and G. Jungck, Common fixed point results for non commuting mappings without continuity in cone metric spaces, J. Math. Anal. Appl. 341(2008), 416-420. 1. 3

[2] I. Beg and M. Abbas, Coincidence point and invariant approximation for mappings satisfying generalized weak contractive condition, Fixed Point Theory Appl. 2006(2006), article ID 74503, 1-7. 1

[3] K. Deimling, Nonlinear Functional Analysis, Springer Verlag, 1985. 1

[4] G.E. Hardy and T.D. Roggers, A generalization of a fixed point theorem of Reich, Canad. Math. Bull. 16 (1973), 201-206. 1

[5] L.G. Huang and X. Zhang, Cone metric spaces and fixed point theorems of contractive mappings, J. Math. Anal.Appl. 332 (2007), 1468-1476. 1. 1. 3

[6] D. Ilic and V. Rakocevic, Common fixed points for maps on cone metric space, J. Math. Anal. Appl. 341 (2008), 876-882. 1

[7] G. Jungck, Commuting maps and fixed points, Amer. Math. Monthly 83 (1976), 261-263. 1

[8] G. Jungck, Compatible mappings and common fixed points, Internat. J. Math. Math. Sci.9 (1986), 771-779. 1 . 1

[9] G. Jungck, Common fixed points for commuting and compatible maps on compacta, Proc. Amer. Math. Soc. 103 (1988), 977-983. 1

[10] G. Jungck, Common fixed points for noncontinous nonself maps on non-metric spaces, Far East J. Math. Sci. 4 (1996), 199-215. 1

[11] G. Jungck and B.E. Rhoades, Fixed points for set valued functions without continuity, Indian J. Pure Appl. Math. 29 (1998), 227-238. 1, 1

[12] R.P. Pant, Common fixed points of noncommuting mappings, J. Math. Anal. Appl.188 (1994), 436-440. 1. 1

[13] S. Rezapour and R. Hamlbarani, Some notes on paper "Cone metric spaces and fixed point theorems of contractive mappings.", J. Math. Anal. Appl. in press. 1, 1, 3

[14] S. Reich, Some remarks concerning contraction mappings, Canad. Math. Bull. 14 (1971),121-124. 1

[15] B.E. Rhoads, A comparison of various definitions of contractive mappings, Trans. Amer. Math. Soc. 26 (1977), 257-290. 1

[16] S. Sessa, On a weak commutativity condition of mappings in fixed point considerations, Publ. Inst. Math. 32 (1982), 149-153. 1, 1

[17] P. Vetro, Common fixed points in cone metric spaces. Rend. Circ. Mat. Palermo 56 (2007), $464-468$.

1

1 Department of Mathematics F. G. Postgraduate College, H-8, Islamabad, 44000, PAKISTAN.

E-mail address: akbarazam@yahoo.com

2 Department of Mathematics, Faculty of Basic and Applied Sciences, InterNational Islamic University, H-10, Islamabad, 44000, Pakistan.

E-mail address: marshad_zia@yahoo.com

${ }^{3}$ Centre for Advanced Studies in Mathematics,Lahore University of Management Sciences, 54792-Lahore, Pakistan

E-mail address: ibeg@lums.edu.pk 